\title{
Incremento del rendimiento y calidad de grano en germoplasma mejorado de trigo (Triticum aestivum L.) del INIAP, en el año 2020
}

Yield increase and grain quality in improved wheat (Triticum aestivum L.) germplasm from INIAP, in 2020

Aumento do rendimento e qualidade dos grãos em germoplasma melhorado de trigo (Triticum aestivum L.) do INIAP, em 2020

\author{
Javier Alberto Garófalo Sosa \\ javier.garofalo@iniap.gob.ec \\ https://orcid.org/0000-0003-2037-5187
}

\author{
Luis Jonatan Ponce-Molina \\ ponceluis75@yahoo.com \\ https://orcid.org/0000-0001-7952-2183
}

\author{
Patricio Javier Noroña Zapata \\ javipatric@hotmail.es \\ https://orcid.org/0000-0003-3930-5883
}

Instituto Nacional de Investigaciones Agropecuarias - INIAP, Estación Experimental Santa Catalina, Programa de Cereales, Ecuador

\section{RESUMEN}

El trigo (Triticum aestivum L.) es uno de los cereales más utilizados por las familias ecuatorianas, con una producción nacional del $1 \%$ de la demanda. El Instituto Nacional de Investigaciones Agropecuarias INIAP, tiene como objetivo generar germoplasma de alto rendimiento y calidad industrial. En el año 2020 en la Estación Experimental Santa Catalina, en parcelas de $3.6 \mathrm{~m} 2$ se evaluaron en campo 580 líneas de trigo seleccionadas durante el 2019 de introducciones provenientes del CIMMYT y de cruzas locales. Los resultados obtenidos muestran que las líneas introducidas alcanzaron un incremento en el rendimiento del $6 \%$ en comparación a los testigos mejorados $(7,14$ t ha-1), entre tanto que, las líneas de cruzas nacionales obtuvieron un incremento del $13,3 \%$. Para la calidad de grano, peso hectolítrico, se observó que las líneas introducidas presentan menores valores de calidad $(71,05 \mathrm{~kg}$ hl-1) en comparación con los testigos (71,87 kg hl-1); mientras que las líneas de cruzas nacionales presentaron un incremento del 2,4\%. Denotando, que los programas de mejoramiento pueden obtener germoplasma con mejores características al realizar sus propios cruzamientos empleando materiales locales y mejor adaptados.

Palabras clave: cruzamientos; fitomejoramiento; genética; líneas; trigo

\section{ABSTRACT}

Wheat (Triticum aestivum L.) is one of the most widely used cereals by Ecuadorian families, with a national production of $1 \%$ of demand. The National Institute of Agricultural and Livestock Researches - INIAP, have the objective of generate germplasm of high yield and industrial quality. In 2020 at the Santa Catalina Experimental Station, 580 wheat lines selected during 2019 from introductions from CIMMYT and local crosses were tested on plots of $3.6 \mathrm{~m} 2$. The results obtained shown that the lines introduced achieved an increase in yield of $6 \%$ compared to the local improved checks (7.14 t ha-1), while the national cross lines obtained an increase of $13.3 \%$. For grain quality, hectolitric weight, it was observed that the lines introduced have lower quality values $(71.05 \mathrm{~kg} \mathrm{hl}-1)$ compared to the control (71.87 kg hl-1); while national cross lines showed an increase of $2.4 \%$. Denoting, that breeding programs can obtain germplasm with better characteristics by making their own crossings using local and better adapted materials.

Key words: breeding, crossing, genetic, lines, wheat

\section{RESUMO}

O trigo (Triticum aestivum L.) é um dos cereais mais utilizados pelas famílias equatorianas, com uma produção nacional de $1 \%$ da procura. O Instituto Nacional de Investigação Agrícola - INIAP, tem por objectivo gerar germoplasma de alto rendimento e qualidade industrial. Em 2020 na Estação Experimental de Santa Catalina, em parcelas de $3,6 \mathrm{~m} 2$, foram avaliadas no campo 580 linhas de trigo seleccionadas durante 2019 a partir de introduções da CIMMYT e cruzamentos locais. Os resultados obtidos mostram que as linhas introduzidas conseguiram um aumento de rendimento de $6 \%$ em comparação com os controlos melhorados (7,14 t ha-1), enquanto as linhas de cruzamentos nacionais obtiveram um aumento de $13,3 \%$. Para a qualidade dos cereais, peso hectolitro, observou-se que as linhas introduzidas apresentaram valores de qualidade inferiores $(71,05 \mathrm{~kg} \mathrm{hl}-1)$ em comparação com os controlos $(71,87$ $\mathrm{kg} \mathrm{hl-1);} \mathrm{enquanto} \mathrm{que} \mathrm{as} \mathrm{linhas} \mathrm{de}$ cruzamentos nacionais apresentaram um aumento de 2,4\%. Isto mostra que os programas de reprodução podem obter germoplasma com melhores características, fazendo os seus próprios cruzamentos utilizando materiais locais e mais adaptados.

Palavras-chave: cruzamentos, genética, linhas, reprodução, trigo 


\section{INTRODUCCIÓN}

El trigo (Triticum aestivum L.) es uno de los cereales de mayor importancia en el mundo. Según la FAO-AMIS se espera que durante el 2021 la producción de trigo se incremente en un $0,69 \%$ a nivel mundial (1). En el año de 2020 Ecuador importó 1'096 589 toneladas de granos de trigo (2) y se sembraron 6498 hectáreas (3); registrándose una productividad por unidad de superficie de $2 \mathrm{t}$ ha- 1 (3), mientras que a nivel mundial la productividad supera las 3,0 t ha-1 (1). En Ecuador, el trigo es cultivado principalmente en la Sierra ecuatoriana entre los 2000 y 3200 metros de altitud, las principales provincias productoras de este cereal son: Carchi, Bolívar, Pichincha, Chimborazo e Imbabura (3).

Las principales estrategias de la mejora del trigo incluyen la introducción de nuevas y novedosas fuentes de variación genética a través de la introducción de germoplasma foráneo (4), el uso de especies silvestres (5), el uso de variedades locales mejor adaptadas $(6,7)$, y el uso de transgenes para caracteres complejos (5).

La introducción de germoplasma es una técnica de mejoramiento muy utilizada en zonas donde los cereales no son endémicos, consiste en colectar o introducir material genético foráneo, esta técnica permite el flujo de nuevo germoplasma aumentando la variabilidad y la introducción de nuevos genes a la región, generalmente se utiliza para mejorar caracteres de altura de planta, resistencia, calidad y producción; esta es una técnica muy empleada por los Programas de Mejoramiento a nivel mundial. (7)
Por otra parte, la mejora tradicional a través de los cruzamientos es el método más empleado para aumentar la variabilidad genética, acumular e introducir nuevos genes; en Ecuador las principales fuentes de variabilidad para la selección de parentales son los materiales locales, lo materiales mejorados nacionales y los materiales introducidos (7). Las variedades tradicionales bien adaptadas a ambientes sometidos a estreses abióticos extremos, ofrecen un reservorio de genes $y$ alelos de especial interés que pueden suponer una mejora en los procesos adaptativos a determinados estreses (8).

Los resultados del fitomejoramiento han sido extraordinarios, especialmente para que a nivel mundial se pueda tener alimento y materias primas para la industria. Ninguna actividad ha sido, es y será tan lucrativa para un país como el mejoramiento genético de plantas y animales. El número de cultivares nacionales disponibles, de una especie determinada, en un país puede ser considerado como medidor del grado de desarrollo de su agricultura, y a medida que esta crece, más cultivares son producidos. Las variedades pasan a tener cada vez más especificidad, más desarrollo y distribución geográfica más estrecha. (9)

La producción de germoplasma adaptado a condiciones específicas requiere de estrategias que permitan la selección en condiciones similares a las del entorno objetivo (favorable o desfavorable) y con implicaciones que se extienden también a elementos de la estrategia de mejoramiento, como la elección de los recursos genéticos y el tipo de variedad (10). 
El objetivo de este trabajo fue analizar MATERIALES Y MÉTODOS el potencial de rendimiento y calidad, de germoplasma proveniente de introducciones y germoplasma de cruzas locales, frente a testigos mejorados.

Tabla 1. Características climáticas del sitio experimental.

\begin{tabular}{|c|c|}
\hline Parámetros & Datos \\
\hline Latitud (S) & $00^{\circ} 22^{\prime} 00^{\prime \prime}$ \\
\hline Longitud (0) & $78^{\circ} 33^{\prime} 17^{\prime \prime}$ \\
\hline Altitud (m.s.n.m) & 3058 \\
\hline Precipitación (mm) & 1408 \\
\hline Temperatura media (oC) & 11.9 \\
\hline
\end{tabular}

El germoplasma utilizado fue semilla proveniente de ensayos internacionales (25 SAWYT, 28 HRWSN, 35 SAWSN, 50 IBWSN, 9 HLBSN, 11 STEMRRSN) provenientes del CIMMYT y poblaciones locales segregantes F7 seleccionados el ciclo 2019. El factor en estudio fue líneas avanzadas de ensayos internacionales y de cruzas locales. La unidad experimental fue una parcela de 3,6 $\mathrm{m}^{2}(3,0 \mathrm{~m}$ $x 1,2 \mathrm{~m})$, conformada por 8 surcos con $15 \mathrm{~cm}$ de separación entre cada uno de estos. Las líneas se sembraron en dos ensayos que se instalaron en campo. No existieron repeticiones. El suelo fue preparado un mes antes de la siembra; utilizando un pase de arado y dos de rastra. La siembra se realizó con una sembradora para experimentos de cereales marca Winterstaiger. La densidad de siembra fue de $180 \mathrm{~kg}$ de semilla ha-1. Previamente, la semilla fue desinfectada con Fludioxonilo en dosis de 2 $\mathrm{ml} \mathrm{kg}^{-1}$ de semilla. Una vez realizado el análisis de suelo, la recomendación de fertilización fue de $80 \mathrm{~kg}$ de $\mathrm{N}, 40 \mathrm{~kg}$ de $\mathrm{P}_{2} \mathrm{O}_{5} \mathrm{ha}^{-1}, 30 \mathrm{~kg}$ de $\mathrm{K}_{2} \mathrm{O}$ $\mathrm{ha}^{-1}, 30 \mathrm{~kg}$ de $\mathrm{S} \mathrm{ha}{ }^{-1}$ y $5 \mathrm{~kg}$ de $\mathrm{Zn} \mathrm{ha-1}$.

Las variables evaluadas fueron: altura de planta, tipo de paja, rendimiento y peso hectolítrico.

\section{RESULTADOS Y DISCUSIÓN}

\section{Altura de planta}

En la Tabla 2, para la variable altura de planta se observó que en los ensayos con líneas internacionales las líneas avanzadas presentaron un promedio de $96,25 \mathrm{~cm}$ frente a los testigos mejorados que presentaron un promedio de 103,50 cm. En cambio, que en los ensayos con líneas de cruzas locales, se observó que el promedio de las líneas avanzadas fue de $109,25 \mathrm{~cm}$ frente a los testigos que presentaron un promedio de $104,68 \mathrm{~cm}$. 
Tabla 2. Estadística descriptiva para altura de planta en líneas avanzadas de ensayos internacionales y cruzas locales.

\begin{tabular}{lcccc}
\hline \multirow{2}{*}{ Parámetro } & \multicolumn{2}{c}{ Ensayos internacionales } & \multicolumn{2}{c}{ Ensayos cruzas locales } \\
& Testigos mejorados & Líneas avanzadas & Testigos mejorados & Líneas avanzadas \\
\hline Número & 30 & 260 & 31 & 259 \\
Promedio $(\mathrm{cm})$ & 103,50 & 96,25 & 104,68 & 109,25 \\
D.E. & 4,38 & 5,97 & 5,76 & 7,75 \\
CV (\%) & 4,24 & 6,2 & 5,51 & 7,09 \\
Mínimo $(\mathrm{cm})$ & 95 & 75 & 95 & 90 \\
Máximo $(\mathrm{cm})$ & 115 & 115 & 115 & 125 \\
\hline
\end{tabular}

Las líneas avanzadas presentaron rangos de altura entre los $75 \mathrm{~cm}$ hasta los $125 \mathrm{~cm}$; en cambio que, que los testigos mejorados presentaron rangos entre los 95 y $115 \mathrm{~cm}$. La mayor variabilidad de valores de altura de planta se observó dentro de las líneas avanzadas (Gráfico 1). Los coeficientes de variación obtenidos estuvieron en el rango de entre 4,38 a $7.75 \%$, considerados buenos y aceptables.

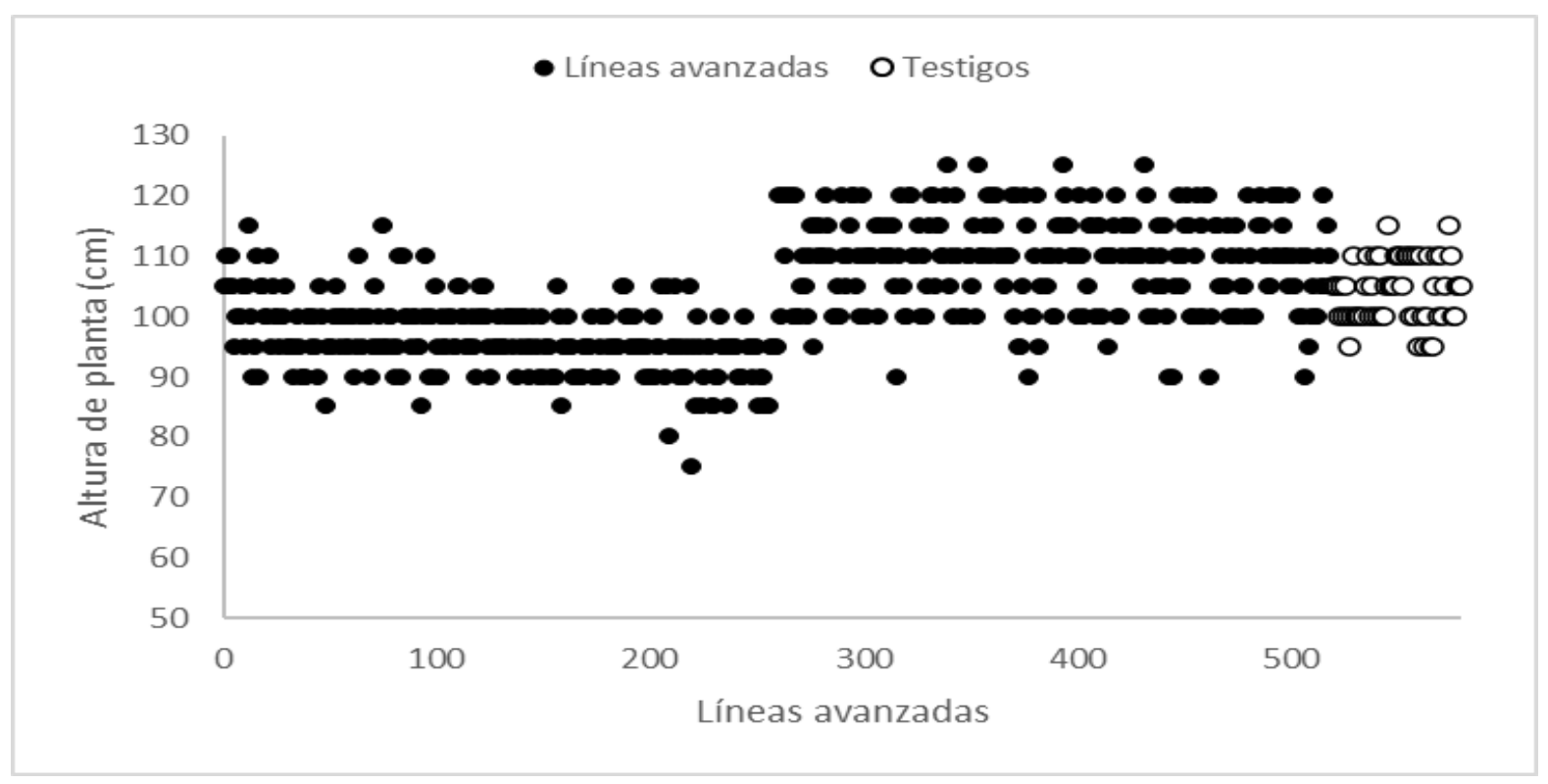

Gráfico 1. Dispersión de datos de altura de planta en población de líneas avanzadas de trigo. 
En la población de líneas avanzadas de ensayos internacionales, se observó dos tipos según la altura de planta, semienano y normal, con una mayoría de plantas de altura normal, 246 individuos. En cambio, en la población de líneas avanzadas de ensayos de cruzas locales, se observó dos tipos según la altura de planta, normal y altos, con una mayoría de altura normal de 214 individuos (Tabla 3).

Tabla 4. Número de líneas avanzadas por tipo según altura de planta.

\begin{tabular}{|c|c|c|c|}
\hline Tipo & Rango $(\mathrm{cm})$ & Ensayos internacionales & Ensayos cruzas locales \\
\hline Enano & $<60$ & 0 & 0 \\
\hline Semienano & $>60 y<90$ & 14 & 0 \\
\hline Normal & $>90 y<120$ & 246 & 214 \\
\hline Alto & $>150$ & 0 & 45 \\
\hline \multicolumn{2}{|c|}{ Total } & 260 & 259 \\
\hline
\end{tabular}

Según Raza et al. (2018) en un estudio realizado en Pakistán donde se comparó líneas mejoradas frente a testigos locales, en la variable altura de planta existió una alta variación entre las diferentes líneas de trigo, donde la máxima altura alcanzada fue de 94,47 $\mathrm{cm}$ y la más baja $84,57 \mathrm{~cm}$, entre tanto que los materiales locales alcanzaron una altura entre 86 y $92 \mathrm{~cm}$. La media general de las líneas fue de $85,5 \mathrm{~cm}$ (11). En cambio, que en un estudio realizado en China por Zhang et al. (2019) donde se comparó 145 líneas introducidas del CIMMYT frente a 7 variedades mejoradas locales, se encontró una alta variación para altura de planta entre las líneas introducidas, donde la máxima altura fue de $101,3 \mathrm{~cm}$ y la más baja 57,20 en el material introducido, entre tanto que en el material mejorado local la altura alcanzada estuvo entre 74 y $90 \mathrm{~cm}$, mientras que la media de todas las líneas (152 líneas) fue de $80,7 \mathrm{~cm}$ (12). Por otra parte, en un estudio realizado en Bolivia se comparó líneas mejoradas frente a un testigo local durante el 2013-2014, y se determinó que para altura de las líneas mejoradas fue de $68,2 \mathrm{~cm}$ a $74,4 \mathrm{~cm}$, mientras que el testigo alcanzó una altura de 70,9 cm (13). Esta información, concuerda con los datos obtenidos en nuestra investigación donde las líneas avanzadas muestran una mayor variación frente a los testigos locales.

\section{Tipo de paja}

La Tabla 4, muestra el tipo de paja que presentaron las líneas avanzadas tanto de los ensayos internacionales y del ensayo de cruzas locales. El promedio general fue de 1.00, correspondiente a líneas con tallo fuerte y resistente al acame. 
Tabla 5. Estadística descriptiva para tipo de paja en líneas avanzadas de ensayos internacionales y cruzas locales.

\begin{tabular}{lcccc}
\hline \multirow{2}{*}{ Parámetro } & \multicolumn{2}{c}{ Ensayos internacionales } & \multicolumn{2}{c}{ Ensayos cruzas locales } \\
& Testigos mejorados & Líneas avanzadas & Testigos mejorados & Líneas avanzadas \\
\hline Número & 30 & 260 & 31 & 259 \\
Promedio & 1,0 & 1,01 & 1.0 & 1,04 \\
D.E. & 0,0 & 0,09 & 0,0 & 0,19 \\
CV (\%) & 0,0 & 8,69 & 0,0 & 18,59 \\
Mínimo & 1 & 1 & 1 & 1 \\
Máximo & 1 & 2 & 1 & 2 \\
\hline
\end{tabular}

En la población de líneas avanzadas de ensayos internacionales, se observó que 258 individuos presentaron tipo de paja de tallo fuerte (1), en cambio que 2 individuos presentaron tipo de paja de tallo intermedio
(2). Para la población de líneas avanzadas de ensayos de cruzas locales, se observó que 249 individuos presentaron tipo de paja 1 tallo fuerte y 10 individuos presentaron tipo de paja 2 tallos intermedios (Tabla 5).

Tabla 6. Número de líneas avanzadas por tipo según tipo de paja.

\begin{tabular}{ccccc}
\hline Tipo & Valor & Ensayos internacionales & Ensayos cruzas locales \\
\hline Tallo fuerte & 1 & 258 & 249 \\
Tallo intermedio & 2 & 2 & 10 \\
Tallo débil & 3 & 0 & 0 \\
& Total & & $\mathbf{2 6 0}$ & $\mathbf{2 5 9}$ \\
\hline
\end{tabular}

Estos resultados concuerdan con los obtenidos por Velasco et al. (2012) que en un estudio realizado en el Valle del Yaqui, Sonora, el $98 \%$ de los genotipos presentaron una resistencia al acame (tallos fuertes), característica fundamental para obtener mayor rendimiento y calidad de grano (14). De igual manera, un estudio realizado en Canadá en líneas de trigo, indica que no existen diferencia estadísticas dentro de la población con respecto al tipo de paja y acame (15).

\section{Rendimiento de grano}

En la Tabla 6, para la variable rendimiento de grano se observa que en el ensayo con líneas avanzadas internacionales estas presentaron un promedio de 7,58 $\mathrm{t} \mathrm{ha}^{-1}$ frente a los testigos mejorados que presentaron un promedio de 7,14 t ha-1. En cambio, que en el ensayo con líneas avanzadas de cruzas locales, se observó que el promedio de las líneas avanzadas fue de $7,33 \mathrm{t}^{-1}$ frente a los testigos que presentaron un promedio de $6,47 \mathrm{t} \mathrm{ha}^{-1}$. 
Tabla 6. Estadística descriptiva para rendimiento de grano en líneas avanzadas de ensayos internacionales y cruzas locales.

\begin{tabular}{lcccc}
\hline \multirow{2}{*}{ Parámetro } & \multicolumn{2}{c}{ Ensayos internacionales } & \multicolumn{2}{c}{ Ensayos cruzas locales } \\
& Testigos mejorados & Líneas avanzadas & Testigos mejorados & Líneas avanzadas \\
\hline Número & 30 & 260 & 31 & 259 \\
Promedio $\left(\mathrm{t} \mathrm{ha}^{-1}\right)$ & 7,14 & 7,58 & 6,47 & 7,33 \\
D.E. & 0,92 & 1,17 & 0,81 & 1,22 \\
CV (\%) & 12,82 & 15,37 & 12,51 & 16,68 \\
Mínimo & 4,89 & 4,11 & 4,06 & 3,11 \\
Máximo & 8,44 & 10,89 & 7,78 & 10,06 \\
\hline
\end{tabular}

Los valores de rendimiento de grano internacionales y de cruzas locales, con obtenidos para las líneas avanzadas coeficientes de desviación (D.E) entre 1.17 presentaron valores entre 3,11 hasta 10,89 y 1.22, respectivamente. Los coeficientes de $\mathrm{t}$ ha $^{-1}$, frente a los testigos que presentaron variación fueron aceptables para este tipo de valores entre 4,06 hasta $8,44 \mathrm{t} \mathrm{ha}^{-1}$ (Gráfico variable evaluada en campo, con valores de 2). La variación de rendimiento de grano fue 12.82 a $16.68 \%$ (Tabla 6).

similar entre las líneas avanzadas de ensayos

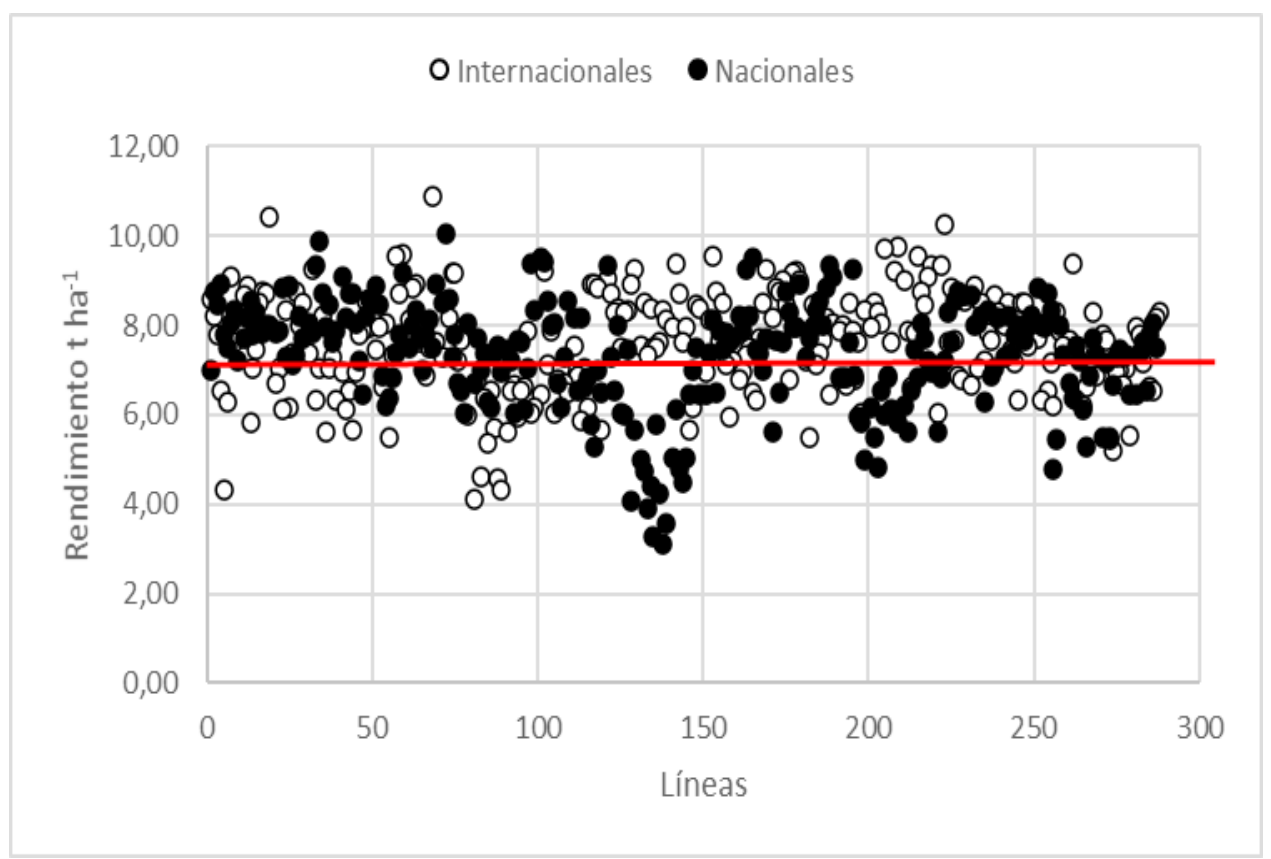

Gráfico 2. Dispersión de datos de rendimiento de grano en población de líneas avanzadas de trigo. 
En la población de líneas avanzadas de ensayos internacionales, se observó que 255 individuos presentaron un rendimiento alto $(>5$ $\mathrm{t} \mathrm{ha-1)}$ y 5 individuos presentaron rendimiento medio ( $>3$ y $<5 \mathrm{t} \mathrm{ha} \mathrm{a}^{-1}$ ). En el ensayo de cruzas locales, se observó que 247 individuos presentaron rendimiento alto y 12 individuos presentaron rendimiento medio (Tabla 5).

Tabla 7. Número de líneas avanzadas por tipo según rango de rendimiento.

\begin{tabular}{ccccc}
\hline Tipo & Rango $\left(\mathbf{t ~ h a}^{-1}\right)$ & Ensayos internacionales & Ensayos cruzas locales \\
\hline Alto & $>5$ & 255 & 247 \\
Medio & $>3-<5$ & 5 & 12 \\
Bajo & $<3$ & 0 & 0 \\
& Total & & $\mathbf{2 6 0}$ & $\mathbf{2 5 9}$ \\
\hline
\end{tabular}

En Pakistán, según Raza et al. (2018), en un estudio donde se comparó líneas mejoradas frente a testigos locales, para el caso de rendimiento también se encontró diferencias en la producción de grano, con un máximo de rendimiento de 5,60 tha ${ }^{-1}$ y un mínimo de 4,60 t ha ${ }^{-1}$ para las líneas introducidas, mientras que los testigos locales alcanzaron un rendimiento de 4,81 a 5,06 t ha-1; entre tanto que la media general del ensayo fue de 4,90 $\mathrm{t} \mathrm{ha}^{-1}$ (11). En la China, Zhang et al. (2019) reporta que para el caso del rendimiento la variación también presentó diferencias, con un máximo de rendimiento de $8,00 \mathrm{tha}^{-1} \mathrm{y}$ un mínimo de 3,87 $\mathrm{t}$ ha ${ }^{-1}$ para el material introducido, mientras que el material mejorado local alcanzó un máximo de 7,64 $\mathrm{t} \mathrm{ha}^{-1}$ y un mínimo de $6,56 \mathrm{t}$ $\mathrm{ha}^{-1}$, y un promedio general de $6,44 \mathrm{t} \mathrm{ha}^{-1}$ (12). En Bolivia, Huanca et al. (2016), registro que las líneas mejoradas alcanzaron un rendimiento entre 2,04 a 3,34 tha-1 $y$ el testigo alcanzó los 2,54 t ha-1 (13). Estos resultados concuerdan con la información obtenida en la presente investigación, y considerando que el rendimiento de grano de las líneas de trigo en un experimento esta mayormente asociado con las condiciones ambientales y su adaptación a estas condiciones $(11,16)$.

\section{Peso hectolítrico}

En la Tabla 8, para la variable peso hectolítrico se observa que en los ensayos con líneas internacionales las líneas avanzadas presentaron un promedio de 71,05 kg hl-1 frente a los testigos mejorados que presentaron un promedio de 71,87 kg hl-1. En cambio, que en los ensayos con líneas de cruzas locales, se observó que el promedio de las líneas avanzadas fue de $73,47 \mathrm{~kg}$ hl-1 frente a los testigos que presentaron un promedio de 71,77 kg hl-1. 
Tabla 8. Estadística descriptiva para peso hectolítrico en líneas avanzadas de ensayos internacionales y cruzas locales.

\begin{tabular}{lcccc}
\hline \multirow{2}{*}{ Parámetro } & \multicolumn{2}{c}{ Ensayos internacionales } & \multicolumn{2}{c}{ Ensayos cruzas locales } \\
& Testigos mejorados & Líneas avanzadas & Testigos mejorados & Líneas avanzadas \\
\hline Número & 30 & 260 & 31 & 259 \\
Promedio $\left(\mathrm{kg} \mathrm{hl}^{-1}\right)$ & 71,87 & 71,05 & 71,77 & 73,47 \\
D.E. & 4,30 & 1,56 & 4,12 & 2,50 \\
CV (\%) & 5,99 & 2,19 & 5,74 & 3,41 \\
Mínimo $\left(\mathrm{kg} \mathrm{hl}^{-1}\right)$ & 66,59 & 65,07 & 66,09 & 67,21 \\
Máximo $\left(\mathrm{kg} \mathrm{hl}^{-1}\right)$ & 76,96 & 74,57 & 76,24 & 79,17 \\
\hline
\end{tabular}

Los valores obtenidos de peso hectolítrico dentro de todas las líneas avanzadas presentaron valores entre 65,07 hasta 79,17 $\mathrm{kg} \mathrm{hl}^{-1}$, frente a los testigos que presentaron valores entre 66,09 hasta 76,96 $\mathrm{kg} \mathrm{hl}^{-1}$ (Gráfico 3). La variación de peso hectolítrico fue similar entre las líneas avanzadas de ensayos internacionales y de cruzas locales, con coeficientes de desviación (D.E) entre 1.56 y 2.50, respectivamente. Los coeficientes de variación fueron aceptables para este tipo de variable evaluada en campo, con valores de 2,19 a 5,99\% (Tabla 9).

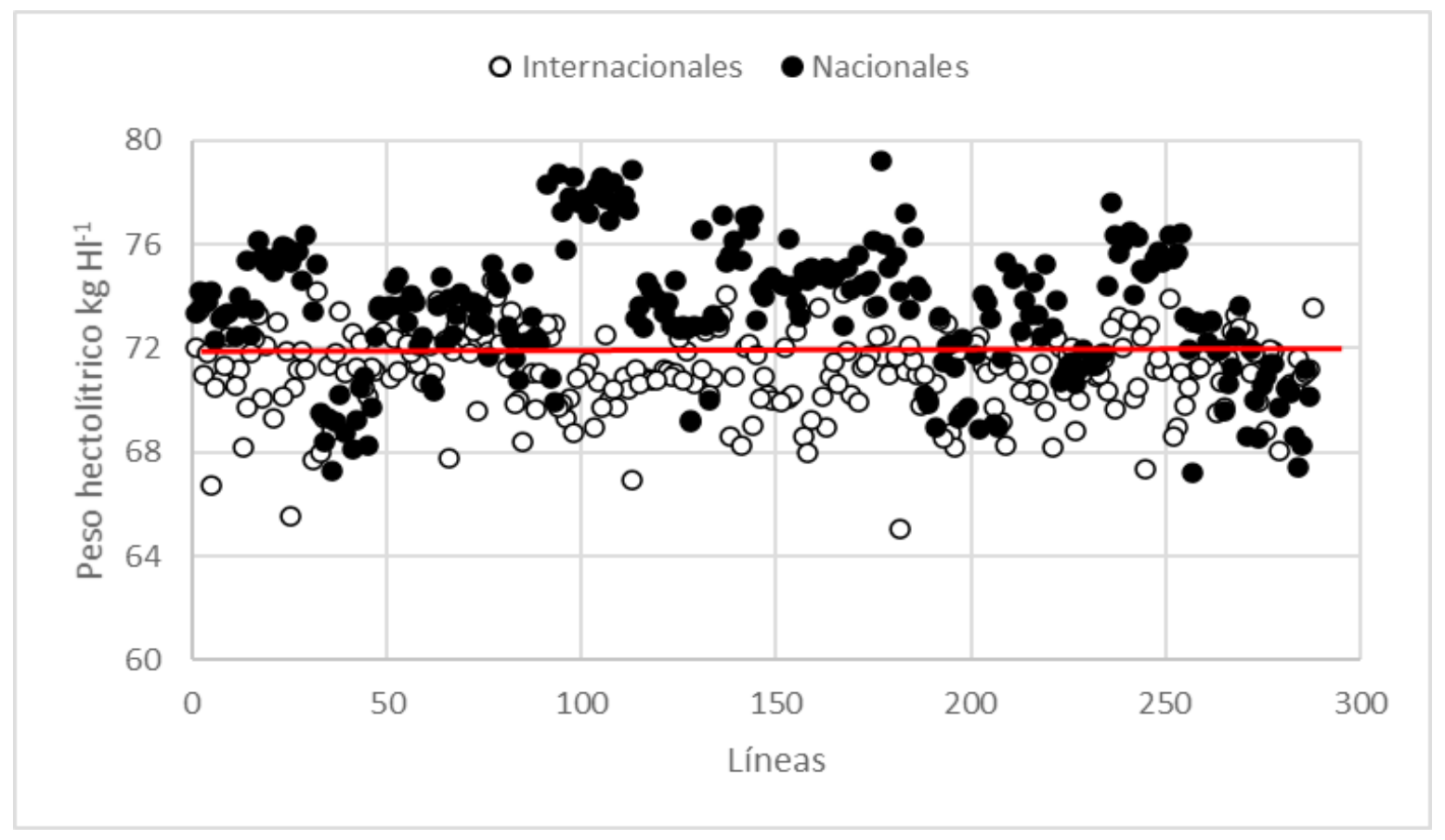

Gráfico 3. Dispersión de datos de peso hectolítrico en población de líneas avanzadas de trigo. 
En la población de líneas avanzadas de ensayos internacionales, se observó que 188 individuos presentaron un peso hectolítrico malo $\left(<72 \mathrm{~kg} \mathrm{hl}^{-1}\right)$ y 72 individuos presentaron peso hectolítrico de bueno y excelente $(>72$ $\mathrm{kg} \mathrm{hl}^{-1}$ ). En el ensayo de cruzas locales, se observó que 181 individuos presentaron un peso hectolitrico bueno y excelente, mientras que solo 68 individuos presentaron un peso hectolítrico malo (Tabla 5).

Tabla 9. Número de líneas avanzadas por tipo según rango de peso hectolítrico.

\begin{tabular}{|c|c|c|c|}
\hline Tipo & Rango (t ha-1) & Ensayos internacionales & Ensayos cruzas locales \\
\hline Excelente & $>74$ & 4 & 112 \\
\hline Bueno & $>72 \mathrm{a}<74$ & 68 & 79 \\
\hline Malo & $<72$ & 188 & 68 \\
\hline \multicolumn{2}{|c|}{ Total } & 260 & 259 \\
\hline
\end{tabular}

En Bolivia, según Huanca et al. (2016), encontró que el peso hectolítrico las líneas mejoradas alcanzaron un peso entre 74,7 y $79,8 \mathrm{~kg} \mathrm{hl}^{-1}$, mientras que el testigo registró un peso de $75,6 \mathrm{~kg} \mathrm{hl}^{-1}$ (13). Resultados que concuerdan con nuestro estudio, en la que los testigos mejorados tienden a tener menor peso hectolítrico que el material mejorado.

En base a los resultados obtenidos de las variables evaluadas se pudo observar que en el ensayo de líneas internacionales (Tabla 10), existieron incrementos positivos para tipo de paja y rendimiento de grano, y negativos para altura de planta y peso hectolítrico; en comparación a los testigos mejorados. La reducción en la altura de planta es un aspecto positivo dentro de los programas de mejoramiento. En este ensayo se seleccionaron un total de 69 líneas, que presentaron características de interés.

Tabla 10. Incremento porcentual por variable evaluada en ensayo de líneas avanzadas de trigo provenientes de ensayos internacionales.

\begin{tabular}{ccccc}
\hline Variable & Testigo & Líneas Internacionales & Incremento & \% Incremento \\
\hline Altura & 103,50 & 96,25 & $-7,30$ & $-7,00$ \\
Tipo de paja & 1,00 & 1,01 & 0,01 & 1,00 \\
Rendimiento & 7,14 & 7,58 & 0,40 & 6,20 \\
Peso hectolítrico & 71,87 & 71,05 & $-0,80$ & $-1,10$ \\
& \multicolumn{2}{c}{ Líneas seleccionadas } \\
\hline
\end{tabular}

Por su parte, en el ensayo de líneas avanzadas con cruzas locales, se observó que existen incrementos positivos en todas las variables evaluadas (Tabla 11 ). Siendo el incremento en rendimiento el valor más alto con $13,30 \%$. El incremento de altura de planta, se considera un aspecto negativo, ya que en los programas de mejoramiento se busca obtener plantas con menor altura. Al final de la evaluación, se seleccionaron 106 líneas que presentaron características de interés. 
Tabla 11. Incremento porcentual por variable evaluada en ensayo de líneas avanzadas de trigo provenientes de ensayos internacionales.

\begin{tabular}{ccccc}
\hline Variable & Testigo & Líneas Internacionales & Incremento & \% Incremento \\
\hline Altura & 104,70 & 109,30 & 4,60 & 4,40 \\
Tipo de paja & 1,00 & 1,04 & 0,04 & 4,00 \\
Rendimiento & 6,50 & 7,30 & 0,90 & 13,30 \\
Peso hectolítrico & 71,80 & 73,50 & 1,70 & 2,40 \\
& \multicolumn{2}{c}{ Líneas seleccionadas } & \\
\hline
\end{tabular}

Reynolds et al. (2007) en un estudio llevado a cabo con más de 2000 entradas de variedades locales mexicanas ensayadas bajo condiciones de estrés abiótico encontró que cerca de una decena mostraron un nivel de adaptación similar a conocidas variedades comerciales, y que el conjunto de variedades locales seleccionadas reveló una amplia diversidad genética, constituyendo un conjunto de variedades de enorme valor para aumentar la base genética de las variedades modernas de trigo adaptadas a estreses abióticos (17).

\section{CONCLUSIONES}

El mayor incremento en las variables evaluadas se observó en las líneas avanzas provenientes de cruzas locales, en comparación con las líneas provenientes de introducciones del CIMMYT.

Dentro de las variables evaluadas, el rendimiento fue la variable con mayor incremento. En las líneas provenientes de las cruzas locales se observó un mayor incremento de rendimiento frente a los testigos.

Para la variable peso hectolítrico, se observó que las líneas nacionales tienen un mejor peso hectolítrico comparados con los testigos, mientras que los materiales introducidos alcanzaron valores inferiores, denotando una menor calidad, esto depende de varios factores, entre ellos, la adaptación a las condiciones agroecológicas locales.

En la variable altura de planta se observó una menor altura en los materiales introducidos. Una menor altura es un aspecto positivo para el mejoramiento.

Los programas de mejoramiento pueden obtener germoplasma con muy buenas características al realizar sus propios cruzamientos empleando materiales locales y mejor adaptados.

\section{REFERENCIAS BIBLIOGRÁFICAS}

1. Market Agricultural Infomation System. Market Monitor [Internet]. 2020. Available from: http://www.amis-outlook.org/\#jfmulticontent_ c363419-2

2. Sistema de Información Pública Agropecuaria SIPA. Principales productos agrícolas [Internet]. 2020. Available from: http://sipa.agricultura. gob.ec/index.php/comercio-exterior

3. Sistema de Información Pública Agropecuaria SIPA. Cifras agroproductivas [Internet]. 2020. Available from: http://sipa.agricultura.gob.ec/ index.php/cifras-agroproductivas

4. Villaseñor Mir HE. Sistema de mejoramiento genético de trigo en México. Rev Mex Ciencias Agrícolas. 2018;(11):2183. 
5. Ortiz R, Trethowan R, Ferrara GO, Iwanaga $M$, Dodds JH, Crouch JH, et al. High yield potential, shuttle breeding, genetic diversity, and a new international wheat improvement strategy. Euphytica. 2007;157(3):365-84.

6. Ortiz-Monasterio J, Sayre K, Rajaram S, McMahon M. Genetic Progress in Wheat Yield and Nitrogen Use Efficiency under Four Nitrogen Rates. Crop Sci [Internet].1997;37(3).

7. Ponce-Molina L, Noroña $P$, Campaña D, Garófalo J, Coronel J, Jiménez C, et al. Generalidades y variedades mejoradas para la Sierra ecuatoriana [Internet]. Primera Ed. Instituto Nacional de Investigaciones Agropecuarias, editor. Quito, Ecuador; 2020. 56 p. Available from: https://repositorio.iniap. gob.ec/bitstream/41000/5587/2/Manual 116 La cebada.pdf.

8. Ruiz de Galarreta Jl, Prohens J, Tierno R. Las variedades locales en la mejora genética de plantas [Internet]. Administración de la Comunidad Autónoma del País Vasco. Departamento de Desarrollo Económico y Competitividad. Vitoria: Eusko Jaurlaritzaren Argitalpen Zerbitzu Nagusia; 2016:465.

9. Vallejo Cabrera FA, Estrada Salazar El. Mejoramiento Genético de Plantas. Universida. Colombia; 2002. 402 p.

10. Ceccarelli S. Specific adaptation and breeding for marginal conditions. Euphytica [Internet]. 1994 Oct;77(3):205-19. Available from: http://link.springer.com/10.1007/ BF02262633

11. Raza A. Evaluation of wheat lines for yield and yield components under rain-fed conditions. Adv Plants Agric Res. 2018;8(6):400-4.
12. Zhang Y, Wang Z, Fan Z, Li J, Gao X, Zhang H, et al. Phenotyping and evaluation of CIMMYT WPHYSGP nursery lines and local wheat varieties under two irrigation regimes. Breed Sci. 2019;69(1):55-67.

13. Huanca N, Quispe G, Marza F. Evaluación de 15 genotipos de trigo harinero ( Triticum aestivum L.), a través de análisis de estabilidad. Rev Boliv [Internet]. 2016;1(8):63-72. Available from: http://www.revistasbolivianas.org.bo/ scielo.php?script=sci_arttext\&pid=S2308250X2016000200010\&lng=es

14. Velasco Magallanes JE, Pérez López $D$ de J, Rajaram Devi S, Balbuena Melgarejo A, Albarrán Mucientes M, González Huerta A. Análisis de 20 genotipos de trigo harinero en el Valle del Yaqui, Sonora. Rev Mex Cienc Agríc. 2012;3:1521-34.

15. Kelbert AJ, Spaner D, Briggs KG, King JR. Screening for lodging resistance in spring wheat breeding programmes. Plant Breed. 2004;123(4):349-54.

16. Porfiri O, Torricelli R, Silveri DD, Papa R, Barcaccia G, Negri V. The Triticeae genetic resources of central Italy: Collection, evaluation and conservation. Hereditas. 2001;135(23):187-92.

17. Reynolds $M$, Dreccer $F$, Trethowan $R$. Drought-adaptive traits derived from wheat wild relatives and landraces. J Exp Bot. 2007;58(2):177-86. 